\title{
Measurement Method for Characterizing Nonlinearity Under Near-Field and Far-Field Interferers in 5G mmW Phased Arrays
}

\author{
Nuutti Tervo, Marko E. Leinonen, Markku Jokinen, Janne Aikio, Olli Kursu, \\ Marko Sonkki, Timo Rahkonen, and Aarno Pärssinen \\ Faculty of Information Technology and Electrical Engineering (ITEE), University of Oulu, Finland
}

\begin{abstract}
A single antenna output of a phased array receiver has only the directivity of the single element. The array gain is achieved once the parallel branches are summed together. The far-field distance of a large array can be several meters which means that for an indoor scenario the interferer can be located in the radiating near-field. In this paper, we present a measurement method for characterizing the nonlinearity of common and parallel paths of the phased array receiver by controlling the number of active $R F$ branches and common branch attenuation. As a measurement platform, we used a 16chain, 64-element phased array receiver operating at $28 \mathrm{GHz}$ center frequency. ACPR is used as a figure of merit of linearity of the receiver and it is measured over-the-air over the azimuth sector by using $100 \mathrm{MHz}$ wide 5GNR CP-OFDM waveform.

Keywords - ACPR, Beamforming, Intermodulation, Spatial interference, over-the-air, 3GPP.
\end{abstract}

\section{INTRODUCTION}

Millimeter-wave $(\mathrm{mmW})$ technologies are emerging in cellular systems and several $\mathrm{mmW}$ bands have already been standardized by third generation partnership project / new radio (3GPP/NR) [1]. In the upcoming years, significant effort is expected especially from industry to put the mmW beamforming systems into practice not only for a fixed link but also for real cellular scenarios with mobile users. As mmW systems become more common in the future, the interference level and potential blockage scenarios are expected to grow in number.

The nonlinearity of a phased array receiver has been studied e.g. in [2] and [3]. The literature has shown that the phase of the nonlinear components in the sum node of the parallel antenna branches depends on the direction of the interference and the beamforming. In [2], it was concluded that the thirdorder intermodulation level (IM3) of a single chain must be specified for maximum received total power (interference + signal), and that using arrays does not provide any benefits from the nonlinearity perspective. However, in practice each nonlinear and linear term observed at the output of parallel receiver chains has a phase progression over the antenna elements which depends on the direction of interference and/or direction of the desired received signal. Hence, the nonlinearity of a receiver array is direction dependent, which makes the measurement scenario challenging to specify.

In general, the receiver gain and linearity budgets are designed such that the nonlinearity of the first RF front-end components does not dominate the distortion. Moreover, the sum node of the phased array has significantly more power compared to the parallel branches which usually makes the common path of the receiver array dominate the nonlinearity.
In order to suppress the interference power at the sum node, zero-forcing and other alike interference reduction techniques and receiver architectures can be used [2], [3]. Furthermore, common path nonlinearity can be naturally controlled by automatic gain control (AGC), e.g. for example by controllable attenuators used in the common branch.

In order to make the parallel chains contribute to the total nonlinearity of the receiver, the interfering users have to (i) have very large transmit effective isotropic radiated power (EIRP), (ii) they must be great in number and/or (iii) the interfering transmitter must be located close to the receiver. High transmit power comes from the highly directive antenna arrays used in the transmission, but in practice the EIRP is limited due to the regulatory requirements [1]. The large number of interferers means that as a sum they provide large enough interference power to have significant impact in the nonlinearity experienced by a single receiver chain. Being close to the receiver can even mean that the user is located in the radiating near-field due to the large aperture of the $\mathrm{mmW}$ phased arrays. For an indoor user, this can easily be a practical scenario as the far-field distance of a decent size antenna array of a base station can be several meters. Indoor access points are usually located in the ceiling or on the wall at a height of 2 - 3 meters and the closest users may be very close to the base station. An example of such scenario is presented in Fig. 1 where the $\mathrm{mmW}$ base station is serving multiple users in a train or in an aircraft.

In this paper, we study the nonlinear impact of a phased array receiver by using $3 \mathrm{GPP} / \mathrm{NR}$ standardized modulated waveforms. Adjacent channel power ratio (ACPR) can be used to characterize receiver nonlinearity as presented in [4]. In the over-the-air (OTA) measurement platform we use 16chain, 64-element phased array operating at $28 \mathrm{GHz}$ center frequency [5]. The transmitter antenna is placed to far-field and radiating near-field and the results are analyzed over the measured azimuth sector of $90^{\circ}$.

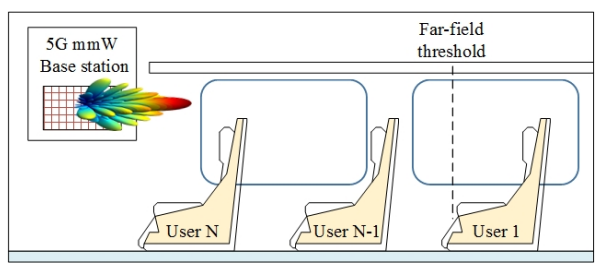

Fig. 1. Potential near-field interference scenario. 


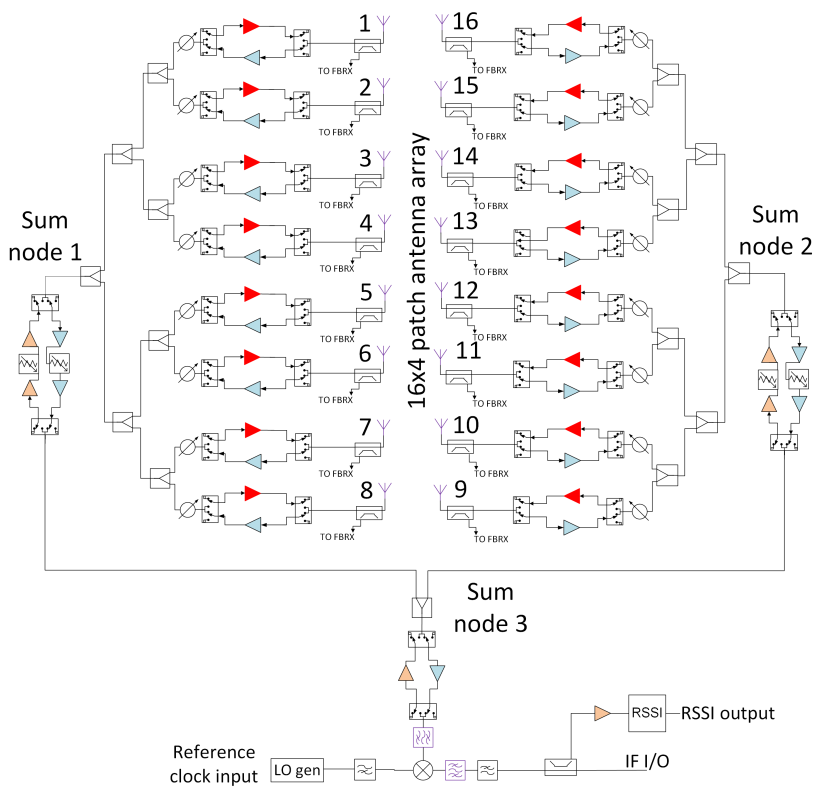

Fig. 2. Overview of the 16-chain, 64-element $28 \mathrm{GHz}$ phased array transceiver.

\section{Demonstration Platform to Characterize the RECEIVER ARRAY NONLINEARITY}

\section{A. 28-GHz Phased Array Receiver Unit}

From the nonlinearity perspective, a phased array receiver can be scarcely divided to parallel paths (from antennas to sum node) and common path (from the sum node all the way to the baseband). In this paper, we use an updated version of 16-chain, 64-element phased array [5]-[6] in which each $\mathrm{RF}$ chain is connected to a dedicated $2 \times 2$ patch element subarray [7]. The block chart of the array is depicted in Fig. 2. The individual antennas are spaced with $\lambda / 2$, leading to $\lambda$-spaced subarrays each followed by a MASW-011036 transceiver (TRx) switch, MAAL-011111 LNA, TGP2100 5bit phase shifter and three PD-0530SM Wilkinson power combiners before the first sum node. Controllable 5-bit HMC939 attenuators are used to control the receiver gain of two stacked 8-chain subarray rows. The rows are summed to one more LNA and HMC264LC3B mixer before the IF output.

In the parallel branches, LNA is assumed to dominate the nonlinearity while in the common branch, there are three LNAs and a mixer which all have impact on the total nonlinearity. In the block chart one should note that there is one LNA between the sum node and the attenuator. Hence, gain control made by the attenuator does not decrease the IM3 caused by the first LNA of the common branch.

\section{B. Over-the-Air Measurement Setup}

An overview of the measurement setup is given in Fig. 3a. Keysight M8190A arbitrary waveform generator (ARB) is used to provide a $100 \mathrm{MHz}$ wide 16-QAM CP-OFDM 5G signal that follows the standard [1]. The signal is upconverted to $28 \mathrm{GHz}$ by E8257B PSG, amplified by CA2630-141 amplifier and fed to A-info LB-28-15 standard gain horn antenna.

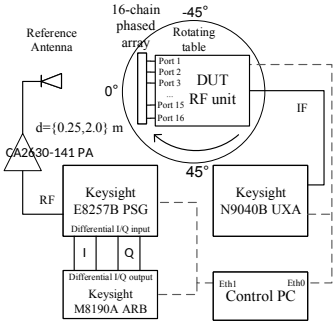

(a)

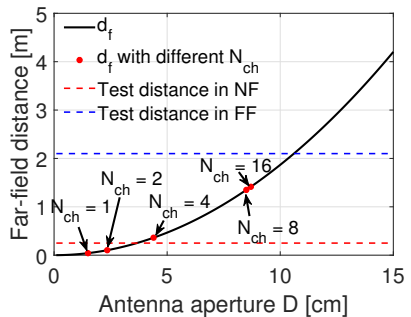

(b)
Fig. 3. (a) Block chart of the measurement setup and (b) Far-field region of the used antenna platform with different configurations.

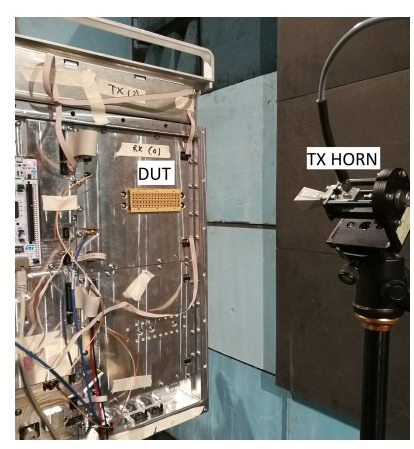

(a)

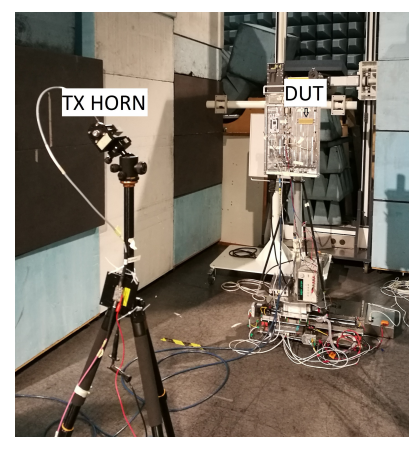

(b)
Fig. 4. Measurement arrangements for (a) near-field and (b) far-field OTA ACPR measurements in EMC-laboratory.

The phased array unit described in II-A is used as the device under test (DUT). The RF center frequency was $28 \mathrm{GHz}$ and the local oscillator (LO) frequency of the DUT was chosen such that the output intermediate frequency (IF) is at $4 \mathrm{GHz}$. The IF output was fed to N9040B UXA signal analyzer in order to measure the channel power $\left(P_{c h}\right)$ and adjacent channel power (ACP). The DUT was placed on top of a stepper motor controlled rotary table and rotated over the azimuth sector of $\theta \in\left[-45^{\circ}, 45^{\circ}\right]$ with $1^{\circ}$ steps. The phase shifters were set to 0 degree (calibrated) in order to disable beamforming in this example. Tx EIRP was set to $20 \mathrm{dBm}$ (RMS) and link distances of $25 \mathrm{~cm}$ (near-field) and $2.1 \mathrm{~m}$ (far-field) were selected. Photographs of the measurement arrangement are depicted in Figs 4a-b.

\section{Near-field versus Far-field}

One of the scenarios where the first LNAs may cause strong contribution to the receiver ACPR is when the interfering transmitter is located in the radiating near-field. In our example phased array, the far-field distance of the whole array is around 1.45 meters. Hence, if the interfering user (in-band or out-of-band) is located closer than this, the impact to the sum node cannot be calculated by the far-field expressions. Yet, it should be concluded that the maximum array gain in the near-field is less than in the far-field. The far field distance can be calculated as

$$
d_{f}=\frac{2 D^{2}}{\lambda_{\min }},
$$


where $D$ is the maximum dimension of the antenna array under test and $\lambda_{\min }$ is the wavelength at the highest frequency component to be measured.

In our array, we can use TRx switch to switch on and off antennas in the receiver. Thus, by controlling the switches of the DUT we can choose the number of active branches from 1 to 16 . The number of active chains has impact on the array nonlinearity for two reasons. First, by increasing the number of active elements we increase the power at the sum node of the parallel branches which in turn increases the power in the common branch. On the other hand, by decreasing the number of antennas, the far-field distance of the array decreases which means that we can bring the transmitter closer and still satisfy the far-field criterion (1). Hence, when measuring the interference source located close to the receiver, we can control the both far-field distance and the power at the sum node by varying the number of active RF branches. The far-field distance is demonstrated in Fig. 3b with different number of active RF branches in the array. Our nearest measurements were done at a distance of $25 \mathrm{~cm}$ in which maximum of 3 branches can be simultaneously active to fulfill the far-field distance (1).

\section{RECEIVER PHASED ARRAY UNDER DIRECTIONAL NEAR- AND FAR-FIELD INTERFERENCE}

\section{A. Nonlinear Beam Patterns}

When specifying the nonlinearity of the parallel receiver branches, it is important to remember that individual antenna elements are blind to array directivity. Hence, the interference is seen in each antenna output, i.e. the first LNA input is only filtered by the individual antenna response, not by the array response. In practice, single elements are not usually very directive due to the large desired steering sector, which means that single element mostly sees the interference with the same gain as the desired signal.

The beam patterns measured over the $25 \mathrm{~cm}$ and $2.1 \mathrm{~m}$ link distances are shown in Fig. 5 for a single element measured in the near-field and in Fig. 6 for the full 16-chain array measured in the far-field. Both channel power and ACP beams are drawn and the difference between the curves is the ACPR. For ACP, we selected the maximum of the lower and upper ACP. The measurement is performed by switching the number of active chains from 1 to 16 with logarithmic steps. By varying the number of active elements we can control the power seen by the sum node and find out which part of the receiver is causing the dominant nonlinearity.

In our system model, we have one LNA at the common path before the attenuator. Hence, we can find out its impact by changing the number of chains and attenuation at the same time. In practice this means that if the number of chains is doubled, we should increase the attenuator by $6 \mathrm{~dB}$. If the ACPR is kept constant by controlling both attenuation and the number of chains simultaneously, we can verify that the first LNA in the sum node is not causing significant nonlinearity to the system.

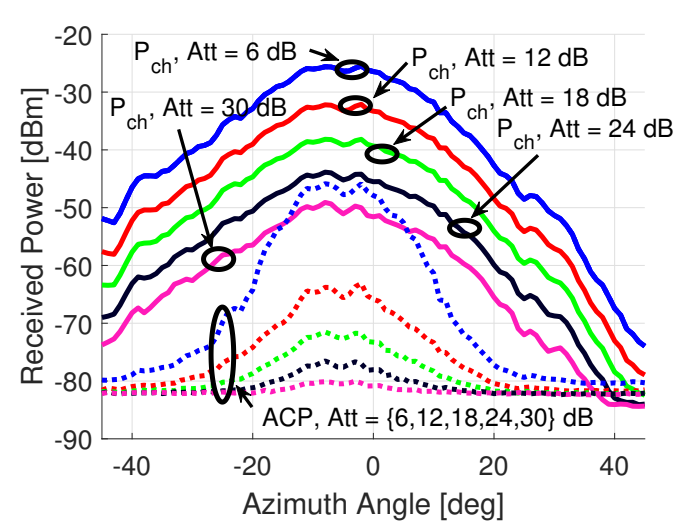

Fig. 5. Channel power and ACP beams measured over the sector for near-field interferer with one active RF chain.

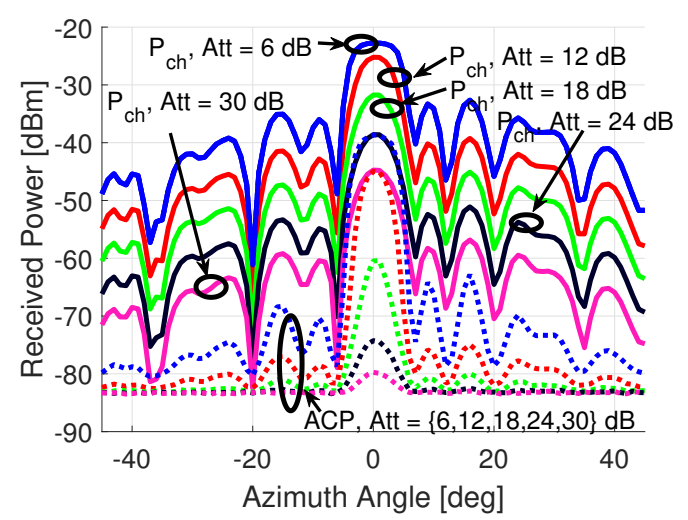

Fig. 6. Channel power and ACP beams measured over the sector for far-field interferer with 16 active RF chains.

\section{B. Worst-case ACPR with Different Antenna Configurations}

The ACPR of the beamforming receiver can be specified as

$$
\mathrm{ACPR}_{\mathrm{Rx}}=\max _{\phi, \theta}(\mathrm{ACP}(\phi, \theta))-\max _{\phi, \theta}\left(P_{\mathrm{ch}}(\phi, \theta)\right)
$$

where $\phi$ and $\theta$ are the azimuth and elevation angles. This can be seen as the worst case which naturally happens when the interference comes from the beamforming direction. It should be noted that if the parallel branches have different nonlinear characteristics due to e.g. different antenna gains, the ACPR varies over the azimuth angle. Also, the noise level contributes to the ACPR when going closer to the noise floor (beam nulls), which limits the angular region to which the ACPR can be characterized. This is why the ACPR of the OTA receiver should be characterized in the beamforming direction.

Figs. 7 and 8 depict the worst-case ACPR with nearfield and far-field interferers and the maximum of those are collected to Figs. 9 and 10. As we can see, for both nearfield and far-field scenario, we can use the number of antenna elements to control the distortion seen at the output of the array. However, in the far field, the ACPR is noise limited with small number of active RF chains. 


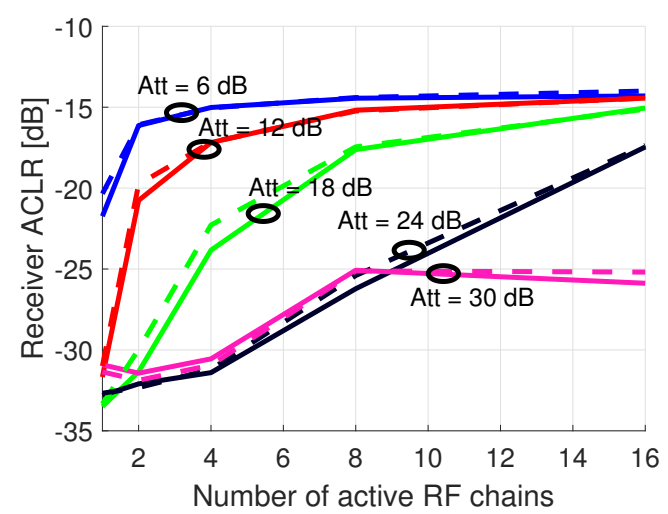

Fig. 7. Maximum ACPR measured over the azimuth sector with near-field interferer.

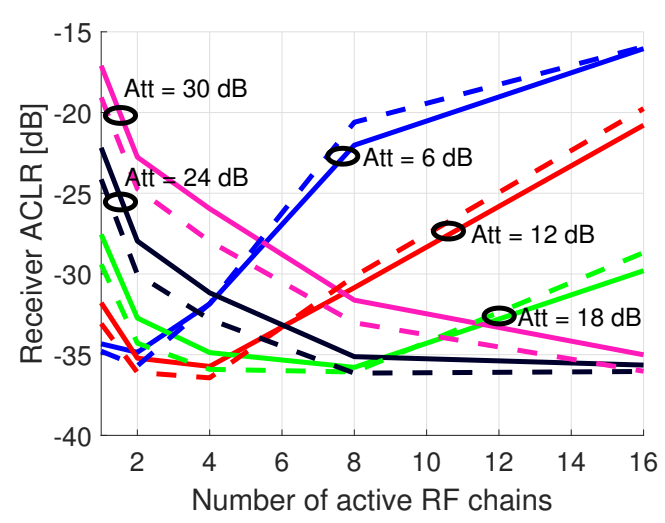

Fig. 8. Maximum ACPR measured over the azimuth sector with far-field interferer.

\section{CONCLUSION}

In this paper, we characterized the nonlinearity of the phased array receiver by controlling the number of active $\mathrm{RF}$ branches. In order to detect the dominant sources of nonlinearity of the array receiver, the number of active RF chains were swept and the nonlinearity in the array output was measured in terms of ACPR by using $100 \mathrm{MHz}$ 5GNR CPOFDM waveform. The measurements were conducted overthe-air by rotating the DUT. As a measurement platform we used 16-chain, 64-element phased array receiver operating at $28 \mathrm{GHz}$ center frequency. We defined the receiver ACPR as the maximum between channel power and adjacent channel power measured over the space. We also showed that the source of interference in realistic $5 \mathrm{G}$ scenario can be in the radiating near-field due to the large aperture of the $\mathrm{mmW}$ arrays. In our measurements, we controlled the far-field distance by changing the number of active elements when the link distance was low. This scenario can be realistic especially indoors where the $5 \mathrm{G}$ $\mathrm{mmW}$ access points are installed to the ceiling or on the wall at a height of couple meters, only.

\section{ACKNOWLEDGMENT}

This research has been supported by the Academy of Finland 6Genesis Flagship (grant no. 318927) and Infotech

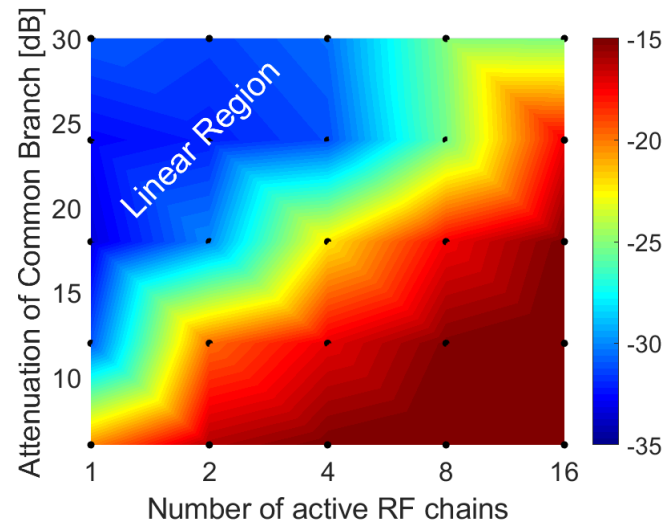

Fig. 9. Maximum ACPR measured with near-field with different antenna configurations and attenuator settings.

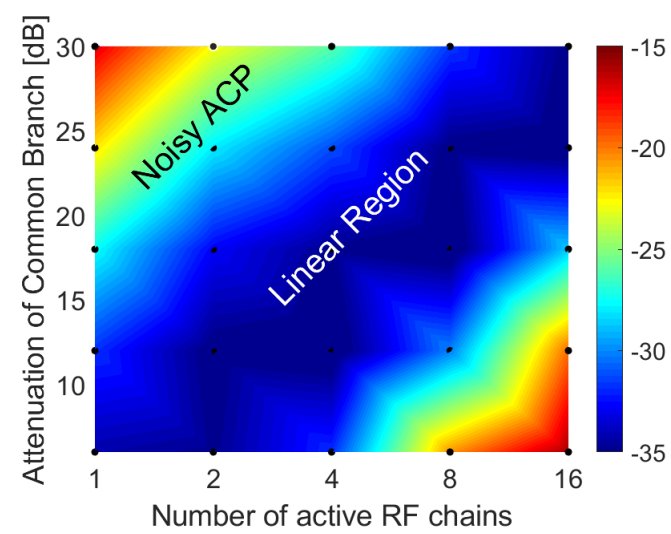

Fig. 10. Maximum ACPR measured with far-field with different antenna configurations and attenuator settings.

Oulu Doctoral Programme.

\section{REFERENCES}

[1] "3rd Generation Partnership Project; Technical Specification Group Radio Access Network; NR; Base Station (BS) radio transmission and reception (Rel 15)." [Online]. Available: http://www.3gpp.org/DynaReport/38series.htm

[2] B. Rupakula and G. M. Rebeiz, "Third-Order Intermodulation Effects and System Sensitivity Degradation in Receive-Mode 5G Phased Arrays in the Presence of Multiple Interferers," IEEE Transactions on Microwave Theory and Techniques, vol. 66, no. 12, pp. 5780-5795, Dec 2018.

[3] M. Soer, E. Klumperink, D. van den Broek, B. Nauta, and F. E. van Vliet, "Beamformer With Constant-Gm Vector Modulators and Its Spatial Intermodulation Distortion," IEEE Journal of Solid-State Circuits, vol. 52, no. 3, pp. 735-746, March 2017.

[4] M. E. Leinonen, N. Tervo, O. Kursu, and A. Pärssinen, "Out-of-Band Interference in 5G mmW Multi-Antenna Transceivers: Co-Existence Scenarios," in 2018 European Conference on Networks and Communications (EuCNC), June 2018, pp. 1-9.

[5] O. Kursu and all., "Design and Measurement of a $5 \mathrm{G} \mathrm{mmW}$ Mobile Backhaul Transceiver at $28 \mathrm{GHz}$," EURASIP Journal on Wireless Communications and Networking, vol. 2018, no. 1, p. 201, Aug 2018.

[6] M. E. Leinonen, G. Destino, O. Kursu, M. Sonkki, and A. Pärssinen, "28 GHz Wireless Backhaul Transceiver Characterization and Radio Link Budget," ETRI Journal, vol. 40, no. 15, pp. 89-100, Feb. 2018.

[7] M. Sonkki and all., "Linearly Polarized 64-element Antenna Array for mm-wave Mobile Backhaul Application," in 12th European Conference on Antennas and Propagation (EuCAP 2018), April 2018, pp. 1-5. 